\title{
ON EFFECTIVE SETS OF POINTS IN RELATION TO INTEGRAL FUNCTIONS*
}

\author{
BY \\ V. GANAPATHY IYER
}

1. Introduction. Let $f(z)$ be an integral function and let $M(r, f)=$ $\max _{|z| \leqq r}|f(z)|$. The order $\rho$ and the type $\kappa(f)$ of $f(z)$ are defined by the relations

$$
\rho=\limsup _{r \rightarrow \infty} \frac{\log \log M(r, f)}{\log r} ; \quad \kappa(f)=\limsup _{r \rightarrow \infty} \frac{\log M(r, f)}{r^{\rho}} .
$$

Let $\left[z_{n}\right], z_{n}=r_{n} e^{i \theta_{n}}$ be a distinct sequence of complex numbers such that

$$
0<r_{1} \leqq r_{2} \leqq \cdots \leqq r_{n} \rightarrow \infty
$$

as $n \rightarrow \infty$. Let $\rho_{1}>0$ be any number. The type $\kappa\left(f, \rho_{1},\left[z_{n}\right]\right)$ of $f(z)$ over the set $\left[z_{n}\right]$ is defined by the relation

$$
\kappa\left(f, \rho_{1},\left[z_{n}\right]\right)=\limsup _{n \rightarrow \infty} \frac{\log \left|f\left(z_{n}\right)\right|}{\left|z_{n}\right| \rho_{1}} .
$$

If $f(z)$ is of order $\rho$, it is evident that $\kappa\left(f, \rho_{1},\left[z_{n}\right]\right) \leqq 0$ when $\rho_{1}>\rho$. If $\rho_{1} \leqq \rho$, the value of $\kappa\left(f, \rho_{1},\left[z_{n}\right]\right)$ can vary from $-\infty$ to $\infty$.

1.1. Definition. Let $f(z)$ be a function of order $\rho$; we shall say that $\left[z_{n}\right]$ is an effective set, or briefly an $E$-set, for $f(z)$ when $\kappa\left(f, \rho,\left[z_{n}\right]\right)=\kappa(f)$.

1.2. It is easy to see that any given function $f(z)$ always possesses an $E$-set; for, on $|z|=r$, there is at least one point $z(r)$ such that $M(r, f)$ $=|f(z(r))|$; also, a sequence $\left[r_{n}\right], r_{1}<r_{2}<\cdots<r_{n} \rightarrow \infty$ as $n \rightarrow \infty$, exists for which

$$
\kappa(f)=\lim _{n \rightarrow \infty} \frac{\log M\left(\boldsymbol{r}_{n}, f\right)}{\boldsymbol{r}_{n}^{\rho}} ;
$$

hence $\left[z\left(r_{n}\right)\right]$ is an effective set for $f(z)$. A more interesting question is to ascertain whether all functions of a given class specified by some simple property possess an $E$-set in common. In this paper an attempt is made to answer this question.

1.3. We denote $C(\rho, d)$ the class of all functions of order $\rho$ and type less than $d$ where $\rho$ and $d$ are any two given positive numbers. We regard all functions of order less than $\rho$ as of order $\rho$ and minimal type, that is $\kappa(f)=0$, un-

\footnotetext{
* Presented to the Society, September 10, 1937; received by the editors January 21, 1937.
} 
less $f(z) \equiv 0$ in which case $\kappa(f)=\kappa\left(f, \rho,\left[z_{n}\right]\right)=-\infty$ for all $\rho$ and $\left[z_{n}\right]$. These are included in $C(\rho, d)$ for the purposes of this paper.

2. We shall, first, discuss a few general properties of an $E$-set for a given class $C(\rho, d)$.

THEOREM 1. In order that a set $\left[z_{n}\right]$ may be an E-set for a class $C(\rho, d)$ it is necessary that

(i) the exponent of convergence (which we shall speak of as the order) of $\left[z_{n}\right]$ cannot be less than $\rho$;

(ii) if the order of $\left[z_{n}\right]$ be $\rho$, any function with zeros at $z=z_{n}$ must be of order $\rho$ and type not less than $d$ unless such a function is identically zero;

(iii) the set $\left[\theta_{n}\right]$ of amplitudes of $\left[z_{n}\right]$ must be everywhere dense in $0 \leqq \theta \leqq 2 \pi$.

Proof. If $\left[z_{n}\right]$ were of order $\rho^{\prime}<\rho$, the canonical product $\sigma(z)$ with simple zeros at $\left[z_{n}\right]$ is of order $\rho^{\prime}$ and therefore is of order $\rho$ and minimal type so that, by the definition of $C(\rho, d)$

But $\sigma\left(z_{n}\right)=0$ so that

$$
\kappa(\sigma)=\kappa\left(\sigma, \rho,\left[z_{n}\right]\right)=0 .
$$

$$
\kappa\left(\sigma, \rho,\left[z_{n}\right]\right)=-\infty .
$$

This contradiction shows that the order of $\left[z_{n}\right]$ cannot be less than $\rho$. A similar argument proves (ii). To prove (iii), suppose that $\left[\theta_{n}\right]$ is not everywhere dense in $(0,2 \pi)$. Then there is a $\theta_{0}$ such that $\theta_{0}-\delta \leqq \theta \leqq \theta_{0}+\delta$ contains no $\theta_{n}, \delta>0$ being sufficiently small. We can suppose without loss of generality that $\theta_{0}=0$ so that the angle $|\theta| \leqq \delta$ does not contain any point of $\left[z_{n}\right]$. Now, let $H_{\rho}(z)$ be defined by*

$$
H_{\rho}(z)=\left\{\begin{array}{lc}
\prod_{n=1}^{\infty}\left(1+\frac{z}{n^{1 / \rho}}\right), & 0<\rho \leqq \frac{1}{2}, \\
\sum_{n=0}^{\infty} \frac{z^{n}}{\Gamma\left(\frac{n}{\rho}+1\right)}, & \rho>\frac{1}{2} .
\end{array}\right.
$$

It is known that, if $z$ is outside $|\theta| \leqq \delta$,

$$
\limsup _{|z| \rightarrow \infty} \frac{\log \left|H_{\rho}(z)\right|}{|z|^{\rho}}<\kappa\left(H_{\rho}\right) .
$$

By considering a function of the form $H_{\rho}(\eta z)$ where $\eta$ is such that $\eta^{\rho} \kappa\left(H_{\rho}\right)<d$, we conclude that $\left[z_{n}\right]$ cannot be an $E$-set for $H_{\rho}(\eta z)$ which obviously belongs to $C(\rho, d)$. Hence $\left[\theta_{n}\right]$ is everywhere dense in $(0,2 \pi)$. It may be noted that

* For $0<\rho \leqq \frac{1}{2}$, see Paley and Wiener, Fourier Transforms in the Complex Domain, p. 79; for $\rho>\frac{1}{2}, H_{\rho}(z)$ are Mittag-Leffler's functions, Acta Mathematica, vol. 29 (1905), pp. 101-181. 
since an $E$-set remains an $E$-set when any other set is added to it, we cannot expect to improve upon the result (i) of Theorem 1.

2.1. We shall now give a general criterion for a set $\left[z_{n}\right]$ to form an $E$-set for a class $C(\rho, d)$. Let $A_{n}(h)$ denote the circle with center $z_{n}$ and radius $\left|z_{n}\right|^{-h}$; and let $A(h)$ denote the system of circles $A_{n}(h), n=1,2, \cdots$. We prove

TheOREM 2. The set $\left[z_{n}\right]$ of order $\rho$ will form an $E$-set for $C(\rho, d)$ provided there exists a function $g(z)$ with simple zeros at $z=z_{n}$ and $h>\rho$ such that the following relations hold:

$$
\lim _{n \rightarrow \infty} \frac{\log \left|g^{\prime}\left(z_{n}\right)\right|}{\left|z_{n}\right|^{\rho}}=d ; \quad \text { (ii) } \quad \lim \frac{\log |g(z)|}{|z|^{\rho}}=d
$$

as $|z| \rightarrow \infty$ outside the circles $A(h)$.

2.2. We shall establish two lemmas in the first place.

LEMma 1. There exists a sequence $\left[R_{n}\right], R_{1}<R_{2}<\cdots<R_{n} \rightarrow \infty$ as $n \rightarrow \infty$, $R_{n+1} \leqq a R_{n}, a>1$ being given, such that no circle $|z|=R_{n}, n=1,2, \cdots$, cuts any circle of $A(h)$.

Proof. Let $b>1$. Consider the ring $r \leqq|z| \leqq b r$. The sum of the diameters of those circles of $A(h)$ whose centers lie in the ring cannot exceed $\sum_{r \leqq\left|z_{n}\right| \leqq b r}\left|z_{n}\right|^{-h}$ which is less than a fixed positive constant since $\sum\left|z_{n}\right|^{-h}$ converges when $h>\rho$. Therefore if $r$ is sufficiently large, there is at least one circle $|z|=R$ in the ring $r \leqq|z| \leqq b r$ which does not cut any circle of $A(h)$. Hence, there is an $n_{0}$ such that for all $n \geqq n_{0}$, the ring $b^{n} \leqq|z| \leqq b^{n+1}$ contains a circle of the type required. Taking $b=a^{1 / 2}$ we get the required result.

Lemma 2. Any function $g(z)$ satisfying the condition (ii) of Theorem 2 is of order $\rho$ and type $d$.

Proof. Let $a>1$ be given and let $\left[R_{n}\right]$ be the sequence of Lemma 1 . On $|z|=R_{n}$, we have, by (ii)

$$
M\left(R_{n}, g\right) \leqq \exp \left[(d+\epsilon) R_{n}^{\rho}\right]
$$

for $n \geqq n_{0}=n_{0}(\epsilon)$. Since $M(r, g)$ is an increasing function of $r$ and $R_{n+1} \leqq a R_{n}$, we get for all $r \geqq r_{0}=r_{0}(\epsilon)$

$$
M(r, g) \leqq \exp \left[a^{\rho}(d+\epsilon) r^{\rho}\right],
$$

so that $\kappa(g) \leqq a^{\rho} d$ and since $a$ is any number greater than one, we get $\kappa(g) \leqq d$. But obviously $d \leqq \kappa(g)$. Hence $\kappa(g)=d$.

2.3. Proof of Theorem 2. Let $f(z)$ be any function of $C(\rho, d)$ and $\left[R_{\nu}\right]$ the sequence of Lemma 1 for some $a>1$. Let 


$$
I_{\nu}=\frac{1}{2 \pi i} \int_{|x|=R_{\nu}} \frac{x^{m} f(x)}{g(x)} \frac{d x}{x-z},
$$

where $m \geqq 0$ is an integer. By (ii) we find that

$$
I_{\nu} \rightarrow 0
$$

as $\nu \rightarrow \infty$ uniformly in any fixed circle $|z| \leqq R$. But

$$
I_{\nu}=\frac{z^{m} f(z)}{g(z)}-\sum_{\left|z_{n}\right|<R_{\nu}} \frac{z_{n}^{m} f\left(z_{n}\right)}{g^{\prime}\left(z_{n}\right)} \frac{1}{z-z_{n}},
$$

while by (i) the series

$$
\sum_{n=1}^{\infty}\left|\frac{z_{n}^{m} f\left(z_{n}\right)}{g^{\prime}\left(z_{n}\right)} \frac{1}{z-z_{n}}\right|
$$

converges uniformly except at the points $z=z_{n}$. Therefore (1) and (2) give

$$
z^{m} \frac{f(z)}{g(z)}=\sum_{n=1}^{\infty} \frac{z_{n}^{m} f\left(z_{n}\right)}{g^{\prime}\left(z_{n}\right)} \frac{1}{z-z_{n}} .
$$

Let $\kappa\left(f, \rho,\left[z_{n}\right]\right)=\beta \leqq \kappa(f)<d$. Choose $\eta$ so that $0<\eta<d-\beta$ and $\lambda$ so that $d \lambda^{\rho}=d-\beta-\eta$. Let $\chi(z)=c_{0}+c_{1} z+c_{2} z^{2}+\cdots$ be any integral function of order $\rho$ and type not exceeding $d \lambda^{\rho}$. Then by (i) the double series

$$
\sum_{(m, n)}\left|\frac{c_{m} z_{n}{ }^{m} f\left(z_{n}\right)}{g^{\prime}\left(z_{n}\right)} \frac{1}{z-z_{n}}\right|
$$

converges uniformly except at $z=z_{n}$, so that (3) gives

$$
\frac{f(z) \chi(z)}{g(z)}=\sum_{n=1}^{\infty} \frac{f\left(z_{n}\right) \chi\left(z_{n}\right)}{g^{\prime}\left(z_{n}\right)} \frac{1}{z-z_{n}} .
$$

In (4) we can take $\chi(z)=g(\lambda z)$ since in this case $\kappa(\chi)=d \lambda^{\rho}$ by Lemma 2 . So (4) gives

$$
f(z)=\frac{g(z)}{g(\lambda z)} \sum_{n=1}^{\infty} \frac{f\left(z_{n}\right) g\left(\lambda z_{n}\right)}{g^{\prime}\left(z_{n}\right)} \frac{1}{z-z_{n}} .
$$

Let $A_{\lambda}(h)$ denote the circles around the zeros of $g(\lambda z)$ similar to $A(h)$. Then, given $a>1$, we can, just as in Lemma 1 , choose a sequence $\left[R_{n}\right], R_{n+1} \leqq a R_{n}$, such that the circles $|z|=R_{n}$ do not cut any circle of either $A(h)$ or $A_{\lambda}(h)$. Using (i), (ii) and the choice of $\lambda$, we get from (5),

$$
M\left(R_{n}, f\right) \leqq \exp \left[\left(d-d \lambda^{\rho}+\epsilon\right) R_{n}^{\rho}\right]
$$

for $n \geqq n_{0}=n_{0}(\epsilon)$. Starting from (6), an argument of the type used in Lemma 2 
shows that $k(f) \leqq d-d \lambda^{\rho}=\beta+\eta$ and since $\eta$ is subject to the sole restriction $0<\eta<d-\beta$ we get $\kappa(f) \leqq \beta$. Since $\beta \leqq \kappa(f)$, we get $\kappa(f)=\beta$ which is the result required.

2.4. In some cases it is possible to conclude that the relation (i) of Theorem 2 follows from (ii). The circles of $A(h)$ determine a sequence of non-overlapping domains $D_{1}, D_{2}, \cdots, D_{n}, \cdots$. Let $p_{n}$ denote the number of points of $\left[z_{n}\right]$ lying in $D_{n}$. We shall prove

LEмma 3. If $p_{n} \leqq P$, a fixed positive number, then (ii) of Theorem 2 involves (i).

Proof. Let $z_{n}$ be contained in $D_{q_{n}}$. Let

and

$$
P_{n}(z)=\prod_{z_{\nu} \subset D_{q_{n}}}\left(1-\frac{z}{z_{\nu}}\right),
$$

$$
g(z)=P_{n}(z) Q_{n}(z) \text {. }
$$

The greatest and the least distances of the boundary of $D_{a_{n}}$ from the origin lie in the interval $\left(\left|z_{n}\right|-H,\left|z_{n}\right|+H\right)$ where $H=\sum_{n=1}^{\infty}\left|z_{n}\right|^{-h}$. Since the degree of $P_{n}(z)$ does not exceed $P$, we have

$$
\lim \frac{\log \left|P_{n}(z)\right|}{|z|^{\rho}}=0,
$$

as $|z| \rightarrow \infty$ outside the domains $D_{\nu}$, uniformly in $n$. Therefore on the boundary of $D_{q_{n}}$, we have by (ii)

(7) $\quad \exp \left[(d-\epsilon)\left(\left|z_{n}\right|-H\right)^{\rho}\right] \leqq\left|Q_{n}(z)\right| \leqq \exp \left[(d+\epsilon)\left(\left|z_{n}\right|+H\right)^{\rho}\right]$.

Since $Q_{n}(z)$ does not vanish in $D_{q_{n}},(7)$ holds in the interior of $D_{q_{n}}$, in particular, at $z=z_{n}$. Hence

Moreover

$$
\lim _{n \rightarrow \infty} \frac{\log \left|Q_{n}\left(z_{n}\right)\right|}{\left|z_{n}\right|^{\rho}}=d
$$

$$
g^{\prime}\left(z_{n}\right)=P_{n}^{\prime}\left(z_{n}\right) Q_{n}\left(z_{n}\right),
$$

and arguing as before we get

$$
\lim _{n \rightarrow \infty} \frac{\log \left|g^{\prime}\left(z_{n}\right)\right|}{\left|z_{n}\right|^{\rho}}=\lim _{n \rightarrow \infty} \frac{\log \left|Q_{n}\left(z_{n}\right)\right|}{\left|z_{n}\right|^{\rho}}=d .
$$

So the lemma is proved.

3. Using Theorem 2, we shall set up an $E$-set for a given class $C(\rho, d)$. We first establish the following 
LeMma 4. Let $\rho=2 / \alpha$ and

$$
\sigma_{\rho}(z)=\prod_{n=1}^{\infty}\left(1-\frac{z^{n}}{n^{\alpha n}}\right) .
$$

Then $\sigma_{\rho}(z)$ is an integral function of order $\rho$ satisfying (i) and (ii) of Theorem 2, with $d=\alpha / 4=1 /(2 \rho)$.

Proof. If we prove (ii) with some $h>\rho$, (i) will follow from Lemma 3 since the circles of $A(h)$ are obviously non-overlapping after a certain stage. Let $p>0$ be any integer and let $z$ lie in the ring $p^{\alpha} \leqq|z| \leqq(p+1)^{\alpha}$.

$$
\sigma_{p}(z)=\left(1-\frac{z^{p}}{p^{\alpha p}}\right)\left(1-\frac{z^{p}}{(p+1)^{\alpha(p+1)}}\right) F_{p}(z),
$$

where

$$
F_{p}(z)=\prod_{n=1}^{p-1}\left(1-\frac{z^{n}}{n^{\alpha n}}\right) \prod_{n=p+2}^{\infty}\left(1-\frac{z^{n}}{n^{\alpha n}}\right)=S_{1} \times S_{2},
$$

say. We have

$$
\begin{aligned}
\log \left|S_{1}\right| & =\frac{1}{2} p(p-1) \log |z|-\alpha \sum_{n=1}^{p-1} n \log n+\log \prod_{n=1}^{p-1}\left|1-\frac{n^{\alpha n}}{z^{n}}\right| \\
& =\frac{\alpha}{4} p^{2}+O(p \log p)+\log \prod_{n=1}^{p-1}\left|1-\frac{n^{\alpha n}}{z^{n}}\right| \\
& =\frac{\alpha}{4}|z|^{2 / \alpha}+O\left(|z|^{1 / \alpha} \log |z|\right)+\log \prod_{n=1}^{p-1}\left|1-\frac{n^{\alpha n}}{z^{n}}\right|
\end{aligned}
$$

Now, since $p^{\alpha} \leqq|z| \leqq(p+1)^{\alpha}$, we have

$$
\prod_{n=1}^{p-1}\left(1-\frac{n^{\alpha n}}{p^{\alpha n}}\right) \leqq \prod_{n=1}^{p-1}\left|1-\frac{n^{\alpha n}}{z^{n}}\right| \leqq \prod_{n=1}^{p-1}\left(1+\frac{n^{\alpha n}}{p^{\alpha n}}\right) .
$$

Using the fact that $(1+1 / x)^{x}$ steadily increases and $(1-1 / x)^{-x}$ steadily decreases to $e$ as $x$ varies in $0<x<\infty$, we get

$$
\begin{aligned}
\left(\frac{n}{p}\right)^{\alpha n} & =\left\{\left(1-\frac{p-n}{p}\right)^{p /(p-n)}\right\}^{\alpha n(p-n) / p} \leqq \exp \left[-\frac{\alpha n(p-n)}{p}\right] \\
& \leqq \exp \left[-\frac{\alpha}{2} \cdot \min (n, p-n)\right] .
\end{aligned}
$$

Therefore (12) gives

$$
0<a=\left\{\prod_{n=1}^{\infty}\left(1-e^{-\alpha n / 2}\right)\right\}^{2} \leqq \prod_{n=1}^{p-1}\left|1-\frac{n^{\alpha n}}{z^{\alpha n}}\right| \leqq\left\{\prod_{n=1}^{\infty}\left(1+e^{-\alpha n / 2}\right)\right\}^{2}=b,
$$


so that by (11),

$$
\log \left|S_{1}\right|=\frac{\alpha}{4}|z|^{2 / \alpha}+O\left(|z|^{1 / \alpha} \log |z|\right) .
$$

A similar argument shows that

$$
\log \left|S_{12}\right|=O(1)
$$

so that (9), (10), and (13) give

$$
\begin{aligned}
\log \left|\sigma_{\rho}(z)\right|= & \frac{\alpha}{4}|z|^{2 / \alpha}+O\left(|z|^{1 / \alpha} \log |z|\right) \\
& +\log \left|1-\frac{z^{p}}{p^{\alpha p}}\right|\left|1-\frac{z^{p+1}}{(p+1)^{\alpha(p+1)}}\right|,
\end{aligned}
$$

where $p^{\alpha} \leqq|z| \leqq(p+1)^{\alpha}$. Taking $h>\rho=2 / \alpha$, we find from (14) that when $z$ is outside the circles of $A(h)$ but inside the ring $p^{\alpha} \leqq|z| \leqq(p+1)^{\alpha}$,

$$
\log \left|\sigma_{\rho}(z)\right|=\frac{\alpha}{4}|z|^{2 / \alpha}+O\left(|z|^{1 / \alpha} \log |z|\right),
$$

and since $p$ is any integer, we get

$$
\lim \frac{\log \left|\sigma_{\rho}(z)\right|}{|z|^{\rho}}=\frac{\alpha}{4}=\frac{1}{2 \rho}
$$

as $|z| \rightarrow \infty$ outside the circles of $A(h)$. So the lemma is proved.

3.1. It is easy to see that if $\left[z_{n}\right]$ is an $E$-set for $C(\rho, d)$ then $\left[\eta z_{n}\right]$ is an $E$-set for $C\left(\rho, d / \eta^{\rho}\right)$. Hence Lemma 4 enables us, in conjunction with Theorem 2 , to state

THEOREM 3. Let $\rho>0, d>0$ be given. The set of points

$$
\left[(2 \rho d)^{-1 / \rho} n^{2 / \rho} e^{2 \nu \pi i / n}\right], \quad n=1,2,3, \cdots ; \nu=0,1,2, \cdots, n-1,
$$

forms an $E$-set for the class $C(\rho, d)$. In other words, if $f(z)$ is any function of order $\rho$ and type less than $d$, then

$$
\limsup _{r \rightarrow \infty} \frac{\log M(r, f)}{r^{\rho}}=\kappa(f)=\limsup _{n \rightarrow \infty} \frac{\log \left|f\left[(2 \rho d)^{-1 / \rho} n^{2 / \rho} e^{2 \nu \pi i / n}\right]\right|}{(2 \rho d)^{-1} n^{2}} .
$$

3.2. As a corollary we get, taking $d=1 /(2 \rho)$, in Theorem 3 ,

THEOREM 4. If $f(z)$ is any integral function of order less than $\rho$ or of order $\rho$ and minimal type $(\kappa(f)=0)$, then

$$
\limsup _{n \rightarrow \infty} \frac{\log \left|f\left(n^{2 / p} e^{2 \nu \pi i / n}\right)\right|}{n^{2}}=0 .
$$


3.3. If $\sigma(z)$ is the canonical product with simple zeros at the lattice points $z=m+i n, m, n=0, \pm 1, \pm 2, \cdots$, it is known from the pseudo-periodic properties of $\sigma(z)$ that (i) and (ii) of Theorem 2 hold for $\sigma(z)$ with $\rho=2$ and $d=\pi / 2$. Hence the class $C(2, \pi / 2)$ has the peculiarly simple $E$-set $z=m+i n, m, n=0, \pm 1, \pm 2, \cdots$.

3.4. I have shown elsewhere* that a function of $C(2, \pi / 2)$ bounded at the lattice-points must be a constant. The question may be asked whether the same is true of an $E$-set for $C(\rho, d)$ for which the conditions of Theorem 2 hold. That this is in fact the case can be shown by using exactly the same method followed in the case of the lattice points. $\dagger$ So we can state

TheOREM 5. Let $\left[z_{n}\right]$ be a set of points satisfying the conditions (i) and (ii) of Theorem 2. Then any function of order $\rho$ and type less than $d$ bounded at the points $\left[z_{n}\right]$ must reduce to a constant.

\subsection{As a particular case of Theorem 5 we get}

THEOREM 6. An integral function of order $\rho$ and type less than $1 /(2 \rho)$ bounded at the points

$$
n^{2 / \rho} e^{2 \nu \pi i / n}, \quad n=1,2,3, \cdots ; \nu=0,1,2, \cdots, n-1
$$

reduces to a constant.

3.6. It may be noted that an $E$-set for a class $C(\rho, d)$ is a fixture to that class and is independent of the individual functions of the class. Theorems 2-4 throw a good deal of light on the peculiar behaviour of the functions of $C(2, \pi / 2)$ at the lattice points. These latter were, in fact, the starting point of the investigations of this paper. It is very probable that conditions closely allied to those of Theorem 2 are also necessary for an $E$-set although I have not succeeded in discovering exactly what these conditions are. The question whether (ii) of Theorem 2 always involves (i) is also unsolved.

* Journal of the London Mathematical Society, vol. 11 (1936), pp. 247-250.

$\dagger$ Since $f\left(z_{n}\right)=0, \kappa(f) \leqq 0$; if $\kappa(f)<0, f(z) \equiv 0$; if $\kappa(f)=0$, then also $\kappa\left(f^{p}\right)=0$, so that formula (3) holds with $m=0$ and $f^{p} / p !, p=0,1,2, \cdots$, in place of $f(z)$. An addition and an argument, as in I.emma 2, will show that $e^{f(z)}$ is of finite order, that is, $f(z)$ is a polynomial which must be a constant since $f\left(z_{n}\right)=O(1)$.

Madras University, MADRAS, S. INDIA 